\title{
Effects of rhBMP-2 Loaded Hydroxyapatite Granules/beta-Tricalcium Phosphate Hydrogel (HA/ $\beta$-TCP hydrogel) Composite on a Rat Model of Coccygeal Intervertebral Fusion.
}

Shinichi Nakagawa

Osaka University Graduate School of Medicine

Rintaro Okada

Minoh Municipal Hospital

Junichi Kushioka

Osaka University Graduate School of Medicine Joe Kodama

Kansai Rosai Hospital

Hiroyuki Tsukazaki

Kansai Rosai Hospital

Zeynep Bal

Osaka University Graduate School of Medicine

Daisuke Tateiwa

Osaka University Graduate School of Medicine

Yuichiro Ukon

Osaka University Graduate School of Medicine

Hiromasa Hirai

Osaka University Graduate School of Medicine

Takahiro Makino

Osaka University Graduate School of Medicine

Shota Takenaka

Osaka University Graduate School of Medicine

Seiji Okada

Osaka University Graduate School of Medicine

Takashi Kaito ( $\nabla$ takashikaito@ort.med.osaka-u.ac.jp )

Osaka University Graduate School of Medicine

\section{Research Article}

Keywords: Hydroxyapatite Granules, Rat Model, Intervertebral Fusion 
Posted Date: November 16th, 2021

DOI: https://doi.org/10.21203/rs.3.rs-1049697/v1

License: @ (i) This work is licensed under a Creative Commons Attribution 4.0 International License. Read Full License 


\section{Abstract}

The effects and inflammation-related side effects of bone morphogenetic protein (BMP)-2 on posterior lumbar interbody fusion are controversial. One of the potential causes for the inconsistent results is the uncontrolled release of BMP-2 from the collagen carrier. Therefore, BMP delivery systems which support effective bone regeneration while attenuating side effects are strongly sought for. We developed NOVOSIS putty (NP), a novel composite material of hydroxyapatite (HA), beta-tricalcium phosphate ( $\beta$-TCP)-hydrogel, and BMP-2, which can sustainably release BMP-2 over two weeks. This study investigated the effects and side effects of NP compared with those of collagen sponge (CS) containing BMP-2 using a rat coccygeal intervertebral fusion model. The fusion rates of NP with low and high doses of BMP-2 were significantly higher than those of an iliac bone (IB) graft, but those of CS with low and high doses of BMP-2 were not different from those of the IB graft. Furthermore, the incidences of ectopic bone formation and soft tissue swelling were significantly lower in the NP group than in the CS group. The HA/ $\beta$-TCP hydrogel carrier enabled superior bone induction with low-dose BMP-2 and decreased the incidence of side effects caused by high-dose BMP-2 compared with that of the collagen carrier.

\section{Introduction}

After the approval of recombinant human bone morphogenetic protein-2 (rhBMP-2) as a bone graft substitute for anterior lumbar intervertebral fusion by the Food and Drug Administration in $2002^{1,2}$, the use of rhBMP-2, including off-label use, has been adopted for approximately $30 \%$ of lumbar spine fusion surgeries in the United States ${ }^{2}$. However, adverse events related to the use of the supraphysiological dose (mg order) of rhBMP-2, such as soft tissue swelling, local inflammation, osteolysis, ectopic bone formation, retrograde ejaculation, and radiculitis, prevent its widespread use $3,4,5$. Thus, there is a need for an efficient drug delivery system for rhBMP-2 to mitigate these adverse events by enabling efficient bone formation with low-dose rhBMP-2.

Several carriers and drug delivery systems have been developed for rhBMP-2 to date, including collagen, hydroxyapatite $(\mathrm{HA})$, beta-tricalcium phosphate ( $\beta-\mathrm{TCP})$, synthetic polymers, and hydrogels ${ }^{6-11}$. HA can provide high mechanical strength, but its biodegradability is limited ${ }^{12}$. $\beta$-TCP is biodegradable, but its mechanical strength is $l o w^{13}$. Synthetic polymers and hydrogels enable sustained release of rhBMP-2 ${ }^{14-16}$; however, a single application of synthetic polymers causes diffculties with molding and handling during surgery. Therefore, we combined multiple carriers (HA/ $\beta$-TCP microsphere/poloxamer 407 hydrogel) and created a novel composite biomaterial for rhBMP-2 (Novosis putty ${ }^{\circledR}$ [NP], CGBio Co. Soul, Korea) to compensate for their shortcomings and take advantage of their strengths. In the NP, HA and $\beta$-TCP are expected to provide scaffolds for new bone formation for long (HA) and short ( $\beta$-TCP) periods, respectively, and the hydrogel would enable sustained release of rhBMP-2 for over three weeks ${ }^{17-19}$.

The purpose of this study was to elucidate the effects of NP as a carrier for rhBMP-2 in bone formation and compare the adverse events of NP with those of a collagen sponge (CS) carrier using a rat coccygeal intervertebral fusion model. 


\section{Results}

\section{Fusion rates}

Rats were divided into the following five groups based on the grafting materials (allogenic iliac bone only [IB group], CS soaked with rhBMP-2 [CS group], and HA/ $\beta$-TCP hydrogel containing rhBMP-2 [NP group]), and the dosage of rhBMP-2 (3 $\mu \mathrm{g}$ of rhBMP-2 [low BMP] or $10 \mu \mathrm{g}$ of rhBMP-2 [high BMP]) $(n=8$ for each group).

Spinal segments harvested at 6 weeks postoperatively were scanned using high-resolution ex vivo microcomputed tomography (micro-CT). Intervertebral fusion was defined as bridging of bone formation on both the coronal and sagittal images of the intervertebral disc space. The fusion rates at the intervertebral disc space were $25 \%$ in the IB group, $50 \%$ in the CS-low BMP group, $62.5 \%$ in the CS-high BMP group, and $87.5 \%$ in both NP-low and -high BMP groups, respectively, as determined using micro-CT. The fusion rate of both the NP groups was significantly higher than that of the IB group $(p=0.04)$. In contrast, the fusion rates of the CS group did not differ from those of the IB group ( $p=0.21)$ (Fig. 1).

Representative coronal micro-CT images of treated intervertebral discs in each group. In the IB group, the grafted bone almost disappeared, and no bridging new bone formation was observed (a). In the CS-low and -high BMP groups, new bone formation that partly bridged the intervertebral disc space was observed, but the bone volume and density were low (b, $c)$. In the NP-low and NP-high groups, the intervertebral disc space was occupied by dense new bone formation with a small amount of HA particles (d, e).

\section{Time-dependent bone changes during the pre-mortal period determined using micro-CT}

In vivo micro-CT was performed immediately after surgery, 2 days postoperatively, and every week after surgery until euthanasia ( 6 weeks). In the IB group, the deisity of the implanted iliac bone decreased with time and the bone almost disappeared at 6 weeks post-operation. In both the CS groups, newly formed bone appeared outside the intervertebral disc space at 2 weeks post-operation, and new bone formation toward the intervertebral disc space was observed; however, the amount of bone formation at the disc space was small even at 6 weeks post-operation. In contrast, in both the NP groups, new bone formation was observed only in the intervertebral space, and the intensity of the new bone increased (Fig. 2).

In the IB group, the grafted bone was absorbed over time and almost disappeared at 6 weeks postoperation (a). In the CS group, new bone formation outside the disc space was observed at 2 weeks postoperation. The amount of new bone formation at the intervertebral disc space was limited until 6 weeks post-operation $(b, c)$. In the NP groups, the density of HA and $\beta$-TCP decreased over time. New bone formation limited to the disc space was observed at 2 weeks post-operation, and the density of the new bone increased over time until 6 weeks post-operation $(d, e)$.

\section{Evaluation of adverse events at the surgical sites}

The incidence of adverse events, including delayed wound healing, soft tissue swelling, ectopic bone formation, and osteolysis, was observed as follows. Ectopic bone formation was diagnosed when bone 
formation outside the intervertebral disc space greater than $2 \mathrm{~mm}$ was observed using in vivo micro-CT (Fig. 3a). Soft tissue swelling, the distance between the skin surface and plate measured using in vivo micro-CT, decreased to less than $1 \mathrm{~mm}$ on day 2 post-operation (Fig. $3 \mathrm{~b}$ ). Delayed wound healing was diagnosed when the operative wound did not heal within 2 weeks (Fig. 3c). Osteolysis of endplates was diagnosed when erosion of the bony endplate $>3 \mathrm{~mm}$ was observed using in vivo micro-CT (Fig. 3d). The total adverse event score (AES) was calculated by assigning one point for each adverse event.

The adverse events at the surgical site are summarized in Table 1. The incidence of ectopic bone formation in the CS-high BMP group (87.5\%) was significantly greater than that in the IB group $(0 \% ; p=0.01)$ and in the NP-high BMP group $(25 \% ; p=0.04)$.

Table1. Summary of the adverse events.

\begin{tabular}{|lllll|}
\hline Group & \multicolumn{2}{l}{$\begin{array}{l}\text { Breakdown of adverse events } \\
\text { Incidence (\%) }\end{array}$} & AES \\
\cline { 2 - 5 } & Ectopic bone & Soft-tissue swelling & Delayed wound healing & \\
\cline { 2 - 5 } & $0^{\#}$ & 12.5 & 12.5 & $0.25 \pm 0.164^{\#}$ \\
IB & $50 *$ & 25 & 25 & $1.0 \pm 0.378$ \\
iliac \\
bone, \\
BMP;
\end{tabular}

rhBMP-2, CS; collagen sponge, NP; Novosis putty, AES; adverse event score, $* ; p<0.05$; (vs. IB group), $\star * ; p<$ 0.001; (vs. IB group), \#; $\mathrm{p}<.05$; (vs. CS-high BMP group)

The incidence of soft tissue swelling was $62.5 \%$ in the CS-high BMP group (62.5\%), 37.5\% in the NP-high BMP group, $25 \%$ in both the CS-low BMP and NP-low BMP groups, and $12.5 \%$ in the IB group. The incidence of soft tissue swelling in the CS-high BMP group was significantly higher than that in the IB group $(p=0.03)$. The incidence of delayed wound healing was not different among the five groups. Osteolysis of the endplates was not observed in this study. AES in the CS-high BMP group (1.88 \pm 0.30$)$ was higher than that in the NP-high BMP group $(0.75 \pm 0.25 ; p=0.04)$.

Operative site (c), and in vivo micro-CT images ( $a, b$, and d) as representative images of adverse events. (a) Ectopic bone formation outside the intervertebral disc space (white arrowhead). (b) Soft tissue swelling. (c) Delayed wound healing. (d) Osteolysis. Double headed arrows represent the measured distances for each event.

\section{Swelling ratio at the surgical sites}


The ratio of soft tissue swelling was calculated by dividing the soft tissue volume (TV) of the surgical site on day 2 post-operation by the TV on one day before surgery (fig.4a). The swelling ratio of the CS-high BMP group $(164.9 \pm 3.4 \%)$ was significantly higher than that of all the other groups. The swelling ratio of the NP groups was not affected by the BMP-2 dose, in contrast to the CS groups, in which the swelling ratio was increased when combined with high-dose BMP-2 (Fig. 4b).

(a) Region of interest for measurement of tissue volume. The dotted line denotes the middle of the intervertebral disc space. (b) Comparison of the swelling ratio among groups. The swelling ratio in the CShigh group was significantly greater than that in the other groups. ${ }^{*} ; p<0.05, * * ; p<0.001$.

\section{Histological analysis}

In the IB group, the intervertebral disc space was predominantly composed of fibrocartilage tissue. In the CS-low BMP group, only a small amount of new bone formation was observed in the intervertebral disc space. In the CS-high BMP group, bridging of new bone formation between the endplates was observed, but the new bone tissue was predominantly composed of adipose tissue. In contrast, in both the NP groups, new bone formation bridging between the endplates was composed of thick trabecular bone. In the NP-low and NP-high groups, a small amount of HA granules remained in the intervertebral disc space, but the majority of $\beta$-TCP disappeared (Fig. 5).

Hematoxylin \& eosin (H\&E) staining of treated segments. Whole intervertebral disc space (a-e), Magnified central parts of the disc space ( $\mathrm{f}-\mathrm{j})$. Fibrous tissue was present between adjacent endplates in the IB group $(a, f)$ and in the CS-low BMP group (b, g). In the CS-high group, new bone formation that bridged the endplates was observed, but the new bone was predominantly composed of adipose tissue $(c, h)$. Thick trabecular bone formation between the endplates was observed in both the NP groups ( $d, e)$. A small amount of HA granules remained inside the newly formed bone $(i, j)$. NB, new bone; HA, hydroxyapatite.

\section{Quantification of the newly formed bone area in the interbody space}

The percentage of new bone area in the region of interest (ROI) was significantly higher in the NP groups (NP-low BMP group, 28.4\%; NP-high group, 30.4\%) than in the CS group (CS-low BMP group, 10.1\%; CS-high group, $15.3 \%$; ${ }^{*}<0.03$; Fig. $6 b$ ).

\section{Discussion}

In this study, the use of HA/ $\beta$-TCP hydrogel composite as a carrier for rhBMP-2 resulted in a superior bone fusion rate and a lower incidence of side effects compared to those following the use of CS in a rat model of coccygeal interbody fusion. In addition, new bone formation in the HA/ $\beta$-TCP hydrogel composite group was localized in the intervertebral disc space; in contrast, ectopic bone formation outside the intervertebral disc space was observed in the group treated with CS. Thus, the use of the HA/ $\beta$-TCP hydrogel composite enabled efficient and spatially controlled new bone formation by rhBMP-2. 
The use of rhBMP-2 remains controversial because the efficacy of BMP-2 for high fusion rate and short operative time may be counteracted by potential side effects such as ectopic bone formation, soft tissue swelling, local inflammation, osteolysis, and retrograde ejaculation ${ }^{4,5}$. In this study, using the HA/ $\beta$-TCP hydrogel as a carrier material for BMP-2, efficient and spatially controlled bone regeneration was achieved with fewer inflammation-related side effects. The following characteristics of the HA/ $\beta$-TCP hydrogel composites are expected to corroborate the results.

The first characteristic of the composite material is the sustained release of rhBMP-2. The BMP-2-related side effects have been reported to be dose dependent ${ }^{20-22}$. Therefore, the decrease in local rhBMP-2 concentration due to sustained release of rhBMP-2 can reduce the side effects. The $\beta$-TCP hydrogel used in this study sustainably releases $20 \%$ of the total rhBMP-2 within 7 days, in contrast to the CS, which releases almost $100 \%$ of the total rhBMP-2 within one day ${ }^{17}$. Another study using the same HA/ $\beta-T C P$ hydrogel composite demonstrated that rhBMP-2 release within 7 days was $19.5 \%$ from the composite and $98.3 \%$ from the collagen carrier ${ }^{19}$. This sustained release of rhBMP-2 is supposed to contribute to the mitigation of BMP-2-related side effects and improvement in fusion rate by controlling the spatial spread of BMP-2 and inducing new bone formation for a long time. In fact, soft tissue swelling did not change between low dose (25\%) and high dose (37.5\%) of rhBMP-2 in the NP groups, in contrast to the CS groups, in which a significant increase in soft tissue swelling was observed in high-dose BMP group (62.5\%) compared to that in low-dose BMP group (25\%). Ectopic bone formation outside the intervertebral space was infrequent in the NP group (low BMP/high BMP, 12.5\%/25\%) than in the CS group (low BMP/high BMP, $50 \% / 87.5 \%)$. In addition, the quality of newly formed bone was superior in the NP group, in which abundant thick trabecular bone formation was observed, than that in the CS group, in which fatty bone marrow was predominant.

Another advantageous characteristic of the HA/ $\beta$-TCP hydrogel composite is the combination of two calcium phosphate scaffolds (HA and $\beta$-TCP) with different biodegradabilities. HA and $\beta$-TCP can provide biomechanical strength and moldability of the composite, and the low-biodegradable HA provides a longterm scaffold for bone formation ${ }^{12}$, and the highly biodegradable $\beta$-TCP microspheres can provide a space for new bone formation in addition to enhancing osteogenic cell differentiation ${ }^{23-25}$. Histological evaluation demonstrated that a small amount of HA granules remained in the intervertebral disc space at 6 weeks post-operation, but the majority of $\beta$-TCP was remodeled and regenerated into new bone (Fig. 5i, j).

This study has several limitations. First, the results for rodent models cannot be directly extrapolated to humans because of the different biomechanics of the spine between quadrupeds and bipeds. Second, no intervertebral fusion cages were applied to the intervertebral disc space in this study. The use of an intervertebral cage might increase the fusion rate in the IB group or rhBMP-2-loaded CS group.

In conclusion, the HA/ $\beta$-TCP hydrogel composite enabled superior bone induction with a low dose of rhBMP-2 and reduced the incidence of side effects caused by high doses of rhBMP-2 compared with the collagen carrier in a rat model of coccygeal intervertebral fusion. The HA/ $\beta$-TCP hydrogel composite is a novel biomaterial for efficient bone regeneration using rhBMP-2. 


\section{Methods}

Characterization of HA and $\beta$-TCP in HA/ $\beta$-TCP hydrogel composite

The HA/ $\beta$-TCP hydrogel biomaterial adopted in this study, which contained $40 \% \mathrm{HA}$ and $60 \% \beta-T C P$, was manufactured by CGBio Co., Ltd., Seongnam, Republic of Korea. In addition to their general appearance, scanning electron microscopy (SEM; Hitachi S-4800, Japan) was used to determine the detailed microstructures of $\beta$-TCP hydrogel and HA (Supplementary Fig. 1).

The HA granules, $\beta$-TCP microspheres, and hydrogel were manufactured by CGBio Co., Ltd., Seongnam, Republic of Korea. The size of HA granules ranged from $3.0 \mathrm{~mm}$ to $6.0 \mathrm{~mm}$, characterized by approximately $70 \%$ of porosity and $99 \%$ of interconnectivity. The $\beta$-TCP microspheres produced using the spray-drying method were $~ 45-75 \mu \mathrm{m}$ in size, with approximately $68 \%$ porosity. The hydrogel was composed of a polyethylene glycol (PEG)/ polypropylene glycol (PPG)/PEG block copolymer and hydroxypropyl methylcellulose (HPMC) composite. The PEG/PPG/PEG block copolymer is thermosensitive, and is null at low temperature and can be mixed homogeneously, while it is gel at body temperature and can be shaped into a desired form. The HPMC composite is viscoelastic, which is helpful for setting a certain shape for the final injected form, with increased resistance against external stress.

\section{Animals and experimental groups}

The Animal Experimental Committee of Osaka University Graduate School of Medicine approved all animal studies (approval number: 28-076-014), which were performed in accordance with ARRIVE guidelines and the National Institutes of Health Guide for the Care and Use of Laboratory Animals ${ }^{30}$. Forty 8-week-old male Sprague-Dawley (SD) rats (Charles River Laboratories, Japan Inc., Kanagawa, Japan) were used in this study. The rats were divided into the following five groups based on the grafting materials: allogenic iliac bone only (IB group), CS soaked with rhBMP-2 (CS group), and HA/ $\beta$-TCP hydrogel containing rhBMP-2 (NP group), as well as the dosage of rhBMP-2 ( $3 \mu \mathrm{g}$ of rhBMP-2 [low BMP] or $10 \mu \mathrm{g}$ of rhBMP-2 [high BMP]) ( $\mathrm{n}=$ 8 for each group) (Table 1). The dosage of rhBMP-2 was decided based on previous reports in which the same coccygeal intervertebral fusion model was used ${ }^{27}$. Volumetric comparison of rat and human intervertebral disc space revealed that $5 \mu \mathrm{g}$ of rhBMP-2 in rats corresponds to $1.5 \mathrm{mg}$ of rhBMP-2 in humans.

\section{Preparation of grafting materials}

\section{Allogenic iliac cancellous bone}

Allogenic iliac cancellous bone was harvested from a donor SD rat immediately before surgery. The volume of iliac bone grafting for each disc space was set to $60 \mathrm{~mm}^{3}(0.05 \mathrm{mg})$.

\section{Collagen sponge containing $\mathrm{hBMP}-2$}


CS (CollaCote; Zimmer Dental, FL, USA) was cut into $5 \mathrm{~mm} \times 5 \mathrm{~mm} \times 3 \mathrm{~mm}\left(6 \mathrm{~mm}^{3}\right)$ sections. Next, $3 \mu \mathrm{g}$ or $10 \mu \mathrm{g}$ of Escherichia coli-derived rhBMP-2 (Daewoong Pharmaceutical Co., Ltd., Seoul, Korea) ${ }^{28}$ was dissolved in phosphate-buffered saline and impregnated into the CS and then freeze-dried (Free Zone 2.5, Labconco Co., Ltd., Kansas city, MO, USA).

\section{HA/ $\beta$-TCP hydrogel composite}

NP consisting of HA/ $\beta-T C P$ microsphere/poloxamer 407 hydrogel was kindly provided by CG Bio Co., Ltd.

28 . The hydrogel contained poloxamer 407 , which is a biodegradable and biocompatible polymer that has been applied in various fields as a biomaterial ${ }^{29,30}$. The hydrogel was mixed with $\beta$-TCP microspheres in a 1:1 weight ratio. Next, HA granules soaked with rhBMP-2 were mixed with the $\beta$-TCP hydrogel in a 3:2 weight ratio in a special mixing syringe to form homogeneous clay-like composites (Fig. 7a). The composites were molded to a volume of $60 \mathrm{~mm}^{3}(0.05 \mathrm{mg})$ before implantation in a container (Fig. $\left.7 \mathrm{~b}\right)$.

\section{Rat coccygeal intervertebral fusion}

All rats were anesthetized using a combination of $0.15 \mathrm{mg} / \mathrm{kg}$ medetomidine (Nippon Zenyaku Kogyo Co., Ltd., Fukushima, Japan), $2.0 \mathrm{mg} / \mathrm{kg}$ midazolam (Astellas Pharma, Inc., Tokyo, Japan), and $2.5 \mathrm{mg} / \mathrm{kg}$ butorphanol (Meiji Seika Pharma Co., Ltd., Tokyo, Japan). Polyoxymethylene plates $(23 \mathrm{~mm} l o n g \times 6 \mathrm{~mm}$ wide $\times 3 \mathrm{~mm}$ high; Matec Co., Ltd., Osaka, Japan) were fixed to the sixth and seventh coccygeal vertebrae through the skin using four stainless steel screws (two screws for each vertebra, $\varphi 1.2 \mathrm{~mm} \times 14 \mathrm{~mm}$; Matsumoto Industry Co., Ltd., Chiba, Japan). The distance between the plates and the skin surface was maintained at $3 \mathrm{~mm}$ at the time of plate fixation (Fig. 7c).

Subsequently, a 10-mm dorsal midline incision was made to expose the intervertebral disc. The posterior one-third of the annulus fibrosus and total nucleus pulposus were removed, and then the cartilaginous endplates were detached and excised using a small raspatory and rongeur to avoid damage to the bony endplates ${ }^{27}$. After irrigation with saline, grafting materials $\left(60 \mathrm{~mm}^{3}\right.$ of IB, CS, or NP) were implanted into the intervertebral disc space (Fig. 7d).

The screw positions and preservation of the bony endplate were confirmed via micro-CT immediately after surgery (Fig. 7e).

Analgesics (2.5 mg/kg butorphanol; Meiji Seika Pharma Co., Ltd., Tokyo, Japan.) and antibiotics $(22,000$ units/kg 1\% penicillin; Meiji Seika Pharma Co., Ltd, Tokyo, Japan.) were subcutaneously administered for 2 postoperative days. Animals were euthanized with carbon dioxide at 6 weeks post-operation, and the operated coccygeal segments were fixed with $10 \%$ formalin.

Preparation of HA/ $\beta$-TCP hydrogel loaded with rhBMP-2 (a). HA/ $\beta$-TCP hydrogel used for insertion (b), coccygeal intervertebral fusion (c), and disc space after implantation of HA/ $\beta$-TCP hydrogel (d). Postoperative sagittal (e, upper) and coronal (e, lower) views of micro-CT, with yellow arrowheads indicating the surface of the bony endplate in the sagittal view. 


\section{In vivo micro-CT analysis}

In vivo micro-CT was performed immediately after surgery, on postoperative day 2 , and every week after surgery until euthanasia ( 6 weeks). The treated coccygeal vertebrae were scanned using micro-CT (R_mCT; Rigaku Mechatronics, Tokyo, Japan) at a resolution of 59 umvoxel in vivo, and the data were collected at $90 \mathrm{kV}$ and $160 \mu \mathrm{A}$. Visualization and data reconstruction were conducted using TRI/3D-BON (Ratoc System Engineering, Tokyo, Japan).

To quantify the soft tissue swelling at the surgical sites, the TV was calculated by setting a ROI on microCT images as follows: a rectangle including the skin surfaces in the axial width and $5 \mathrm{~mm}$ in the longitudinal length that centers the intervertebral disc space (threshold [ $L=15500]$, software; TRI/3D-BON, Ratoc System Engineering, Tokyo, Japan) (Fig. 4a). The ratio of soft tissue swelling was calculated by dividing the TV of the surgical site on postoperative day 2 by the TV one day before surgery.

\section{Ex vivo micro-CT analysis}

Spinal segments harvested at 6 weeks post-operation were scanned using high-resolution ex vivo micro-CT (Skyscan 1272, Bruker, Belgium). The scanning parameters were as follows: camera binning $=2 \times 2$, source voltage $(k V)=80$, source current $(\mu A)=125$, image pixel size $(\mu \mathrm{m})=10$, rotation step (degree) $=1.0$, and filter = Al $1 \mathrm{~mm}$. Image analysis was performed using CTAN software (Version 1.18.8.0+, Bruker, Belgium).

\section{Histological analysis}

Dissected and formalin-fixed coccygeal segments were fixed with $10 \%$ formic acid, dehydrated in a graded ethanol series, decalcified with K-CX (Falma, Tokyo, Japan), and embedded in paraffin wax. Serial sagittal sections (3- $\mu \mathrm{m}$ thickness) were cut and stained with H\&E. A $1.5 \times 2-\mathrm{mm}^{2} \mathrm{ROI}$ (interbody space) was extracted from the newly formed fusion mass. The new bone area (red) was color coded using ImageJ software (version 1.52q, U. S. National Institutes of Health, Bethesda, Maryland, USA) ${ }^{31}$, and the percentage of the newly formed bone area in the ROI was calculated (Fig. 6a).

\section{Statistical analysis}

All statistical analyses were performed using the JMP 15 Statistics software (SAS Institute Inc., Cary, NC, USA). Results are presented as means \pm standard deviation. Fisher's exact test was used to compare the success of fusion and the incidence of adverse events in each group. Differences in the measured variables between multiple groups were analyzed using one-way analysis of variance followed by Dunnett's test or Tukey-Kramer test. Statistical significance was set at $p<0.05$.

\section{Declarations}

\section{Acknowledgments}

This study was funded by Nippon Zoki Pharmaceutical Co., Ltd. NP was generously provided by CGBio Co., Ltd. 
Author Contributions Statement

TK and SN designed this study. SN, DT, and RO contributed to the animal surgery. SN, DT, RO, J. Kodama, J. Kushioka, HT, BZ, YU, HH, ST, TM, SO and TK interpreted and analyzed the data. SN wrote the manuscript, and TK critically reviewed it. All authors have read and approved the final manuscript.

\section{Competing Interests}

The authors declare no competing interest.

\section{References}

1 Burkus JK, Gornet MF, Dickman CA, Zdeblick TA. Anterior lumbar intervertebral fusion using rhBMP-2 with tapered intervertebral cages. J Spinal Disord Tech. 2002 Oct;15(5):337-49.

2 Ong KL, Villarraga ML, Lau E, Carreon LY, Kurtz SM, Glassman SD. Off-label use of bone morphogenetic proteins in the United States using administrative data. Spine (Phila Pa 1976). 2010 Sep 1;35(19):1794800 .

3 Cahill KS, Chi JH, Day A, Claus EB. Prevalence, complications, and hospital charges associated with use of bone-morphogenetic proteins in spinal fusion procedures. JAMA. 2009 Jul 1;302(1):58-66.

4 Hustedt JW, Blizzard DJ. The controversy surrounding bone morphogenetic proteins in the spine: a review of current research. Yale J Biol Med. 2014 Dec 12;87(4):549-61.

5 Tannoury CA, An HS. Complications with the use of bone morphogenetic protein 2 (BMP-2) in spine surgery. Spine J. 2014 Mar 1;14(3):552-9.

6 Ohura K, Bohner M, Hardouin P, Lemaître J, Pasquier G, Flautre B. Resorption of, and bone formation from, new beta-tricalcium phosphate-monocalcium phosphate cements: an in vivo study. J Biomed Mater Res. 1996 Feb;30(2):193-200.

7 Dong J, Uemura T, Shirasaki Y, Tateishi T. Promotion of bone formation using highly pure porous betaTCP combined with bone marrow-derived osteoprogenitor cells. Biomaterials. 2002 Dec;23(23):4493-502.

8 Chazono M, Tanaka T, Komaki H, Fujii K. Bone formation and bioresorption after implantation of injectable beta-tricalcium phosphate granules-hyaluronate complex in rabbit bone defects. J Biomed Mater Res A. 2004 Sep 15;70(4):542-9.

9 Walsh WR, Vizesi F, Michael D, Auld J, Langdown A, Oliver R, Yu Y, Irie H, Bruce W. Beta-TCP bone graft substitutes in a bilateral rabbit tibial defect model. Biomaterials. 2008 Jan;29(3):266-71.

10 Tanaka T, Komaki H, Chazono M, Kitasato S, Kakuta A, Akiyama S, Marumo K. Basic research and clinical application of beta-tricalcium phosphate ( $\beta$-TCP). Morphologie. 2017 Sep;101(334):164-172. 
11 Kakuta A, Tanaka T, Chazono M, Komaki H, Kitasato S, Inagaki N, Akiyama S, Marumo K. Effects of micro-porosity and local BMP-2 administration on bioresorption of $\beta$-TCP and new bone formation. Biomater Res. 2019 Jul 26; 23: 12.

12 Araújo M, Linder E, Lindhe J. Effect of a xenograft on early bone formation in extraction sockets: an experimental study in dog. Clinical oral implants research. 2009 Jan;20(1):1-6. Epub 2009/01/08.

13 Sanda M, Shiota M, Fujii M, Kon K, Fujimori T, Kasugai S. Capability of new bone formation with a mixture of hydroxyapatite and beta-tricalcium phosphate granules. Clinical oral implants research. 2015 Dec;26(12):1369-74. Epub 2014/08/27.

14 Kaito T, Myoui A, Takaoka K, Saito N, Nishikawa M, Tamai N, Ohgushi H, Yoshikawa H. Potention of the activity of bone morphogenetic protein- 2 in bone regeneration by a PLAPEG/hydroxyapatite composite. Biomaterials 2005; 26: 73-79

15 Miyamoto S, Takaoka K, Okada T, Yoshikawa H, Hashimoto J, Suzuki S, Ono K. Evaluation of polylactic acid homopolymers as carriers for bone morphogenetic protein. Clinical Orthopaedics and Releated Research 1992; 287: 274-285 14

16 Saito N, Takaoka K. New synthetic biodegradable polymers as BMP 1 carriers for bone tissue engineering. Biomaterials 2003; 24: 2287-2293

17 Lee JH, Kim J, Baek HR, Lee KM, Seo JH, Lee HK, Lee AY, Zheng GB, Chang BS, Lee CK. Fabrication of an rhBMP-2 loaded porous $\beta$-TCP microsphere-hyaluronic acid-based powder gel composite and evaluation of implant osseointegration. J Mater Sci Mater Med. 2014 Sep;25(9):2141-51.

18 Lyu HZ, Lee JH. The efficacy of rhBMP-2 loaded hydrogel composite on bone formation around dental implants in mandible bone defects of minipigs. Biomater Res. 2020;24:5.

19 Tateiwa D, Nakagawa S, Tsukazaki H, Okada R, Kodama J, Kushioka J, Zeynep B, Ukon Y, Hirai H, Kaito T. A novel BMP-2 loaded hydroxyapatite/beta-tricalcium phosphate microsphere/hydrogel composite for bone regeneration. Scientific Reports. 2021 in press.

20 Boakye M, Mummaneni PV, Garrett M, Rodts G, Haid R. Anterior cervical discectomy and fusion involving a polyetheretherketone spacer and bone morphogenetic protein. J Neurosurg Spine. 2005 May;2(5):521-5.

21 Shields LB, Raque GH, Glassman SD, Campbell M, Vitaz T, Harpring J, Shields CB. Adverse effects associated with high-dose recombinant human bone morphogenetic protein-2 use in anterior cervical spine fusion. Spine (Phila Pa 1976). 2006 Mar 1;31(5):542-7.

22 Lee KB, Taghavi CE, Song KJ, Sintuu C, Yoo JH, Keorochana G, Tzeng ST, Fei Z, Liao JC, Wang JC. Inflammatory characteristics of rhBMP-2 in vitro and in an in vivo rodent model. Spine (Phila Pa 1976). 2011 Feb 1;36(3): E149-54. 
23 LeGeros RZ, Lin S, Rohanizadeh R, Mijares D, LeGeros JP. Biphasic calcium phosphate bioceramics: preparation, properties and applications. Journal of materials science Materials in medicine. 2003 Mar;14(3):201-9. Epub 2004/09/07.

24 Habibovic P, Sees TM, van den Doel MA, van Blitterswijk CA, de Groot K. Osteoinduction by biomaterials--physicochemical and structural influences. Journal of biomedical materials research Part $A$. 2006 Jun 15;77(4):747-62. Epub 2006/03/25.

25 Yuan H, Kurashina K, de Bruijn JD, Li Y, de Groot K, Zhang X. A preliminary study on osteoinduction of two kinds of calcium phosphate ceramics. Biomaterials. 1999 Oct;20(19):1799-806. Epub 1999/10/06.

26 National Research Council Committee for the Update of the Guide for the, C. \& Use of Laboratory, A. in Guide for the Care and Use of Laboratory Animals (National Academies Press (US) Copyright (C) 2011, National Academy of Sciences., 2011).

27 Okada R, Kaito T, Ishiguro H, Kushioka J, Otsuru S, Kanayama S, Bal Z, Kitaguchi K, Hashimoto K, Makino T, Takenaka S, Sakai Y, Yoshikawa H. Assessment of effects of rhBMP-2 on intervertebral fusion with a novel rat model. Spine J. 2020 May;20(5):821-829.

28 Lee JH, Jang SJ, Koo TY, Seo CW, Lee KM, Lee HS, Baek HR. Expression, Purification and Osteogenic Bioactivity of Recombinant Human BMP-2 Derived by Escherichia Coli. Tissue Engineering and Regenerative Medicine. 2011 Vol. 8, No. 1, pp 8-15.

29 Lee JH, Ryu MY, Baek HR, Seo JH, Lee KM, Lee JH. Generation of an rhBMP-2-loaded beta-tricalcium phosphate/hydrogel composite and evaluation of its efficacy on peri-implant bone formation. Biomed Mater. 2014 Aug 18;9(5):055002.

30 Lee JH, Ryu MY, Baek HR, Lee HK, Seo JH, Lee KM, Lee AY, Zheng GB, Chang BS, Lee CK. The effects of recombinant human bone morphogenetic protein-2-loaded tricalcium phosphate microsphere-hydrogel composite on the osseointegration of dental implants in minipigs. Artif Organs. 2014 Feb;38(2):149-58.

31 Schneider, C. A., Rasband, W. S. \& Eliceiri, K. W. NIH Image to ImageJ: 25 years of image analysis. Nature methods 9, 671-675, doi:10.1038/nmeth.2089 (2012).

\section{Figures}

IB

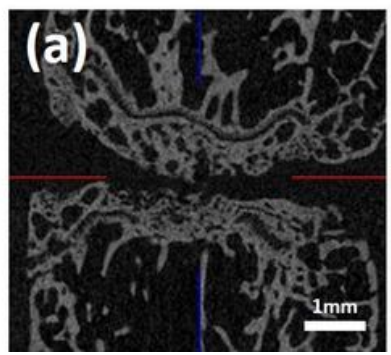

CS-low BMP

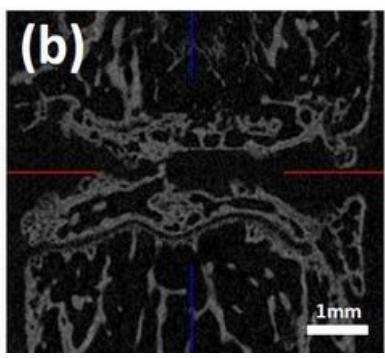

CS-high BMP

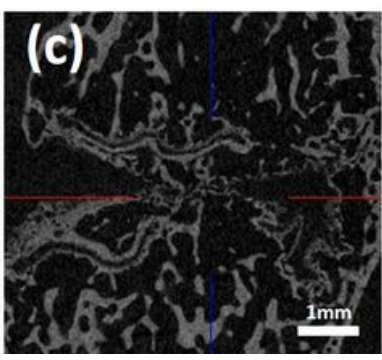

Page 13/18
NP-low BMP
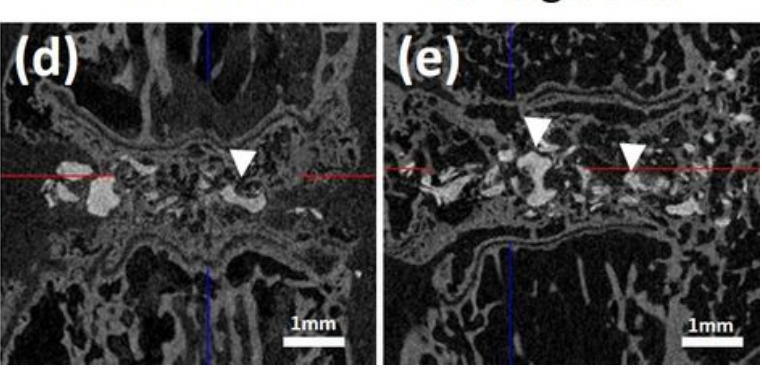

NP-high BMP 
Figure 1

Ex vivo micro-CT images of treated segments

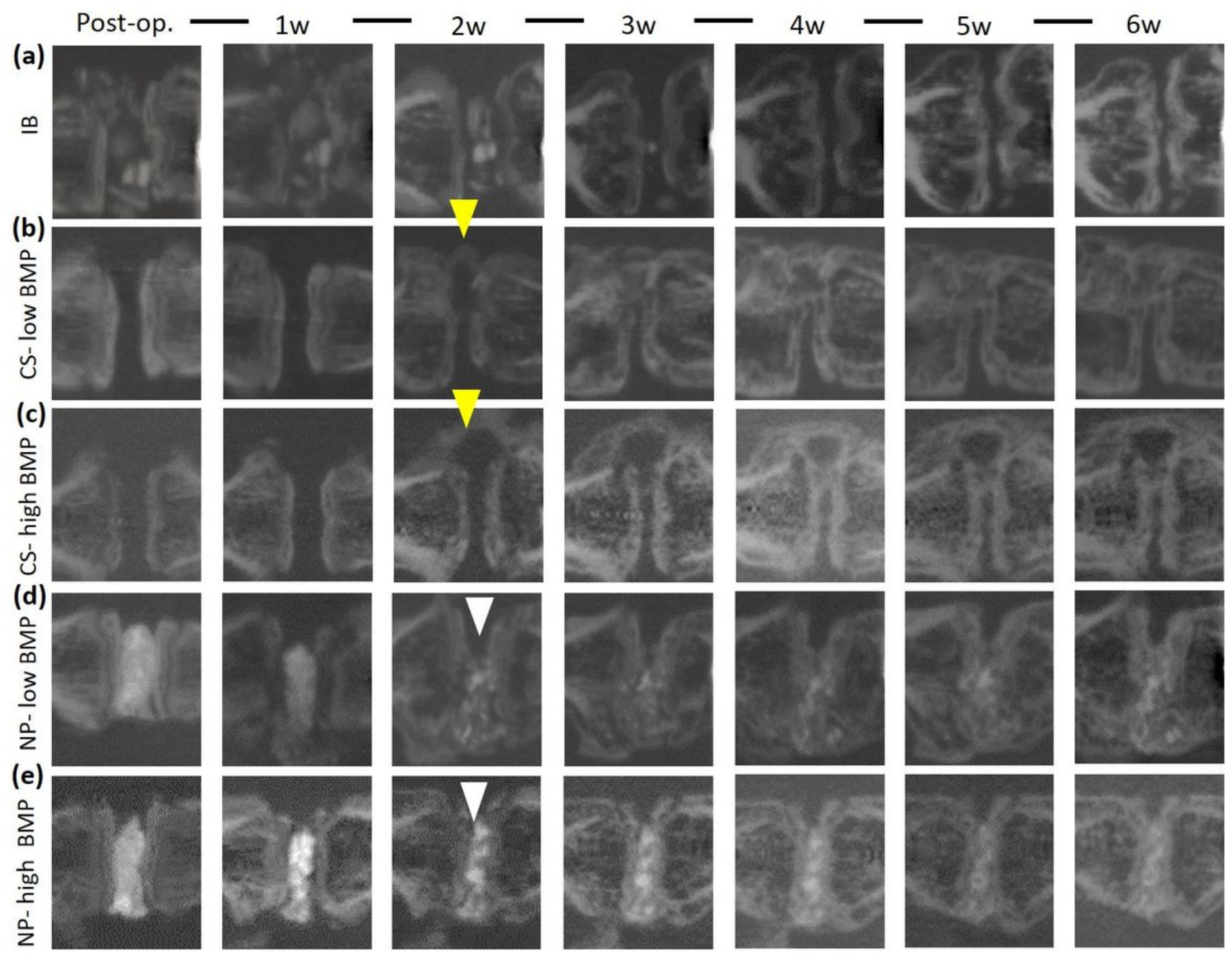

Figure 2

Temporal in vivo micro-CT images of the treated segments 
(a)

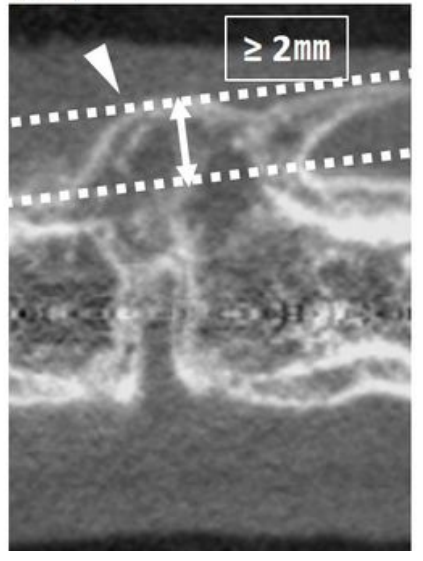

(b)

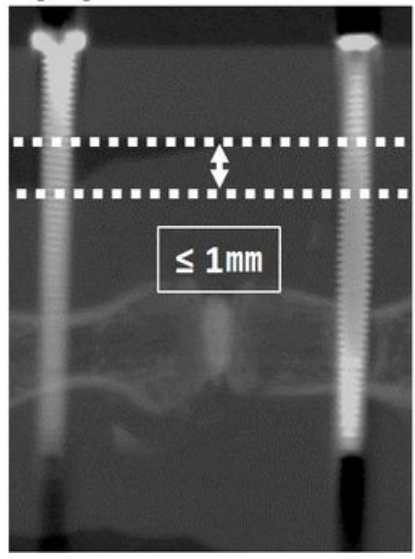

(c)

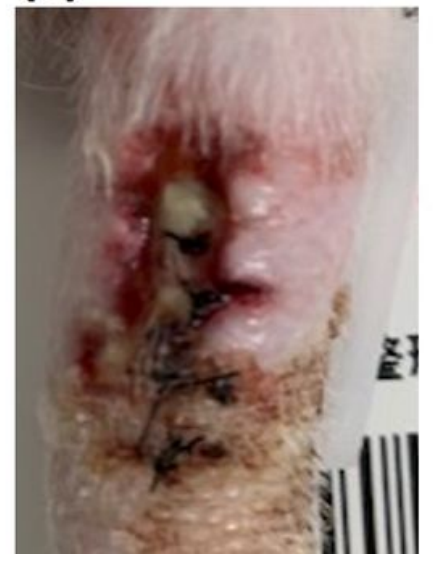

(d)

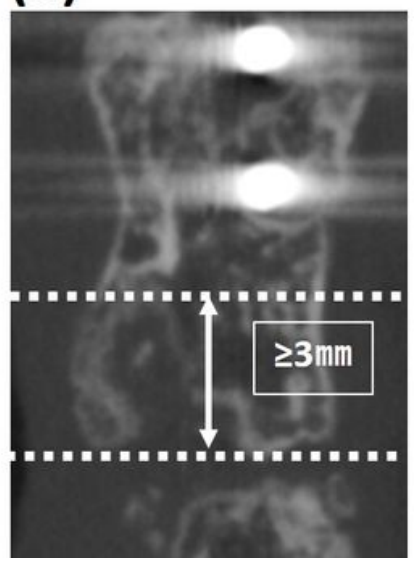

Figure 3

Representative images of each event.

(a)

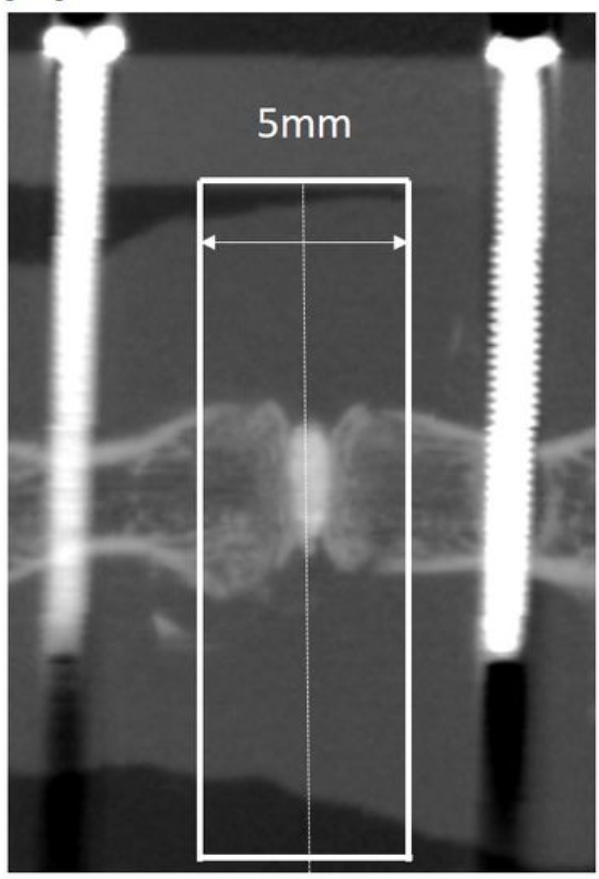

(b)

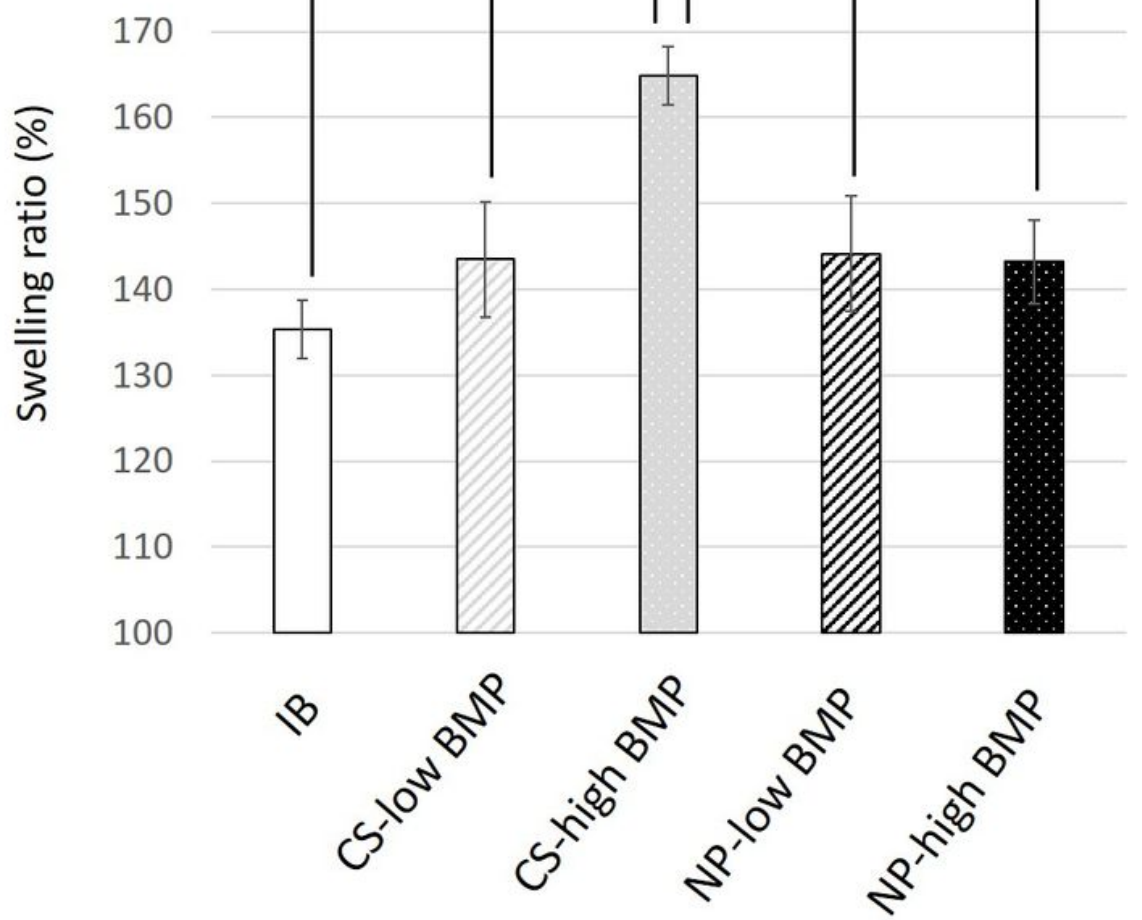

Figure 4

Quantification of surgical site swelling. 

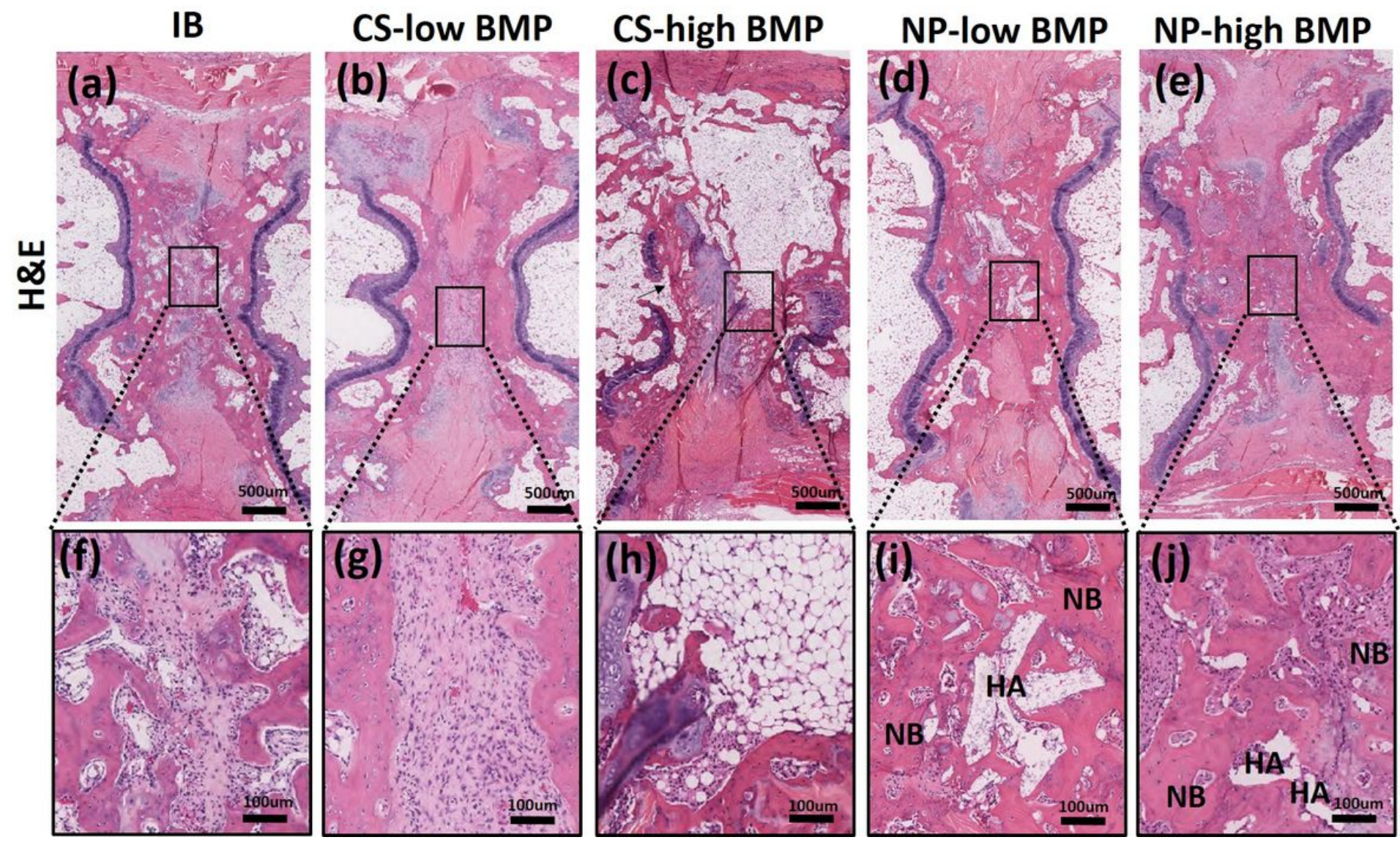

Figure 5

Histological images of treated segments at 6 weeks post-operation. 
(a)

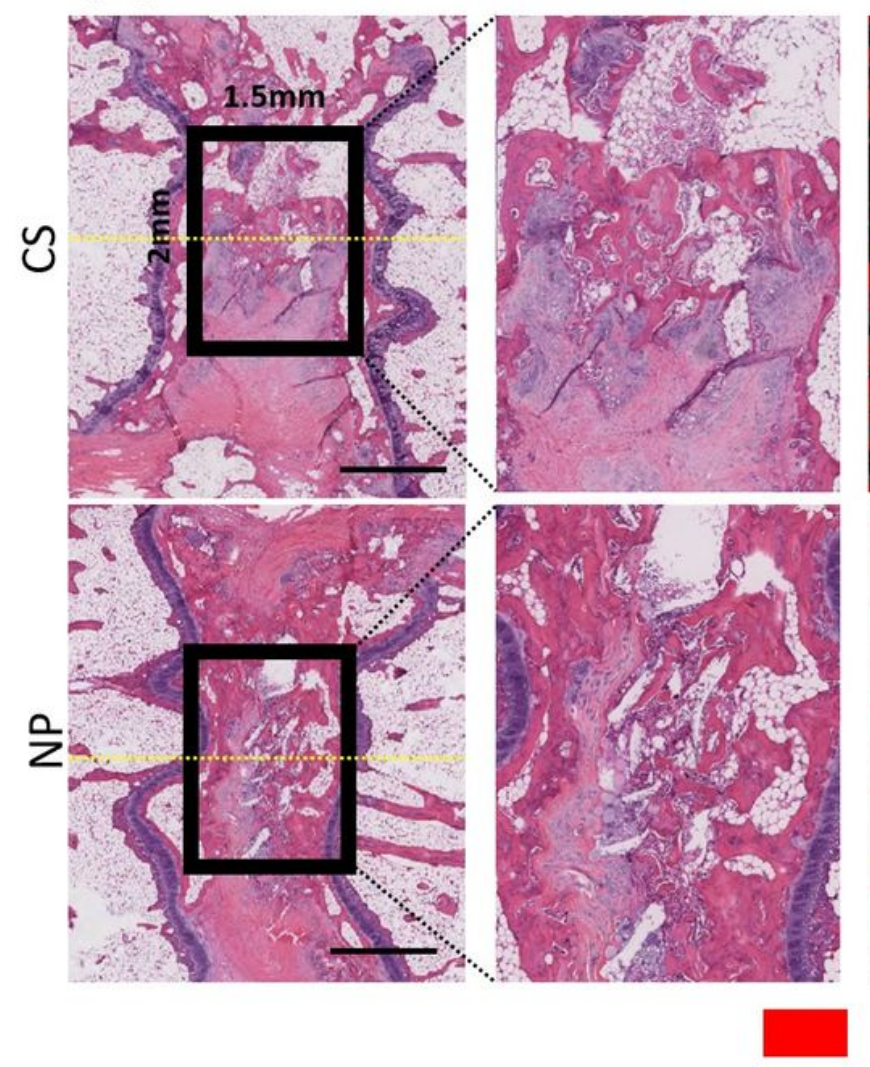

(b)
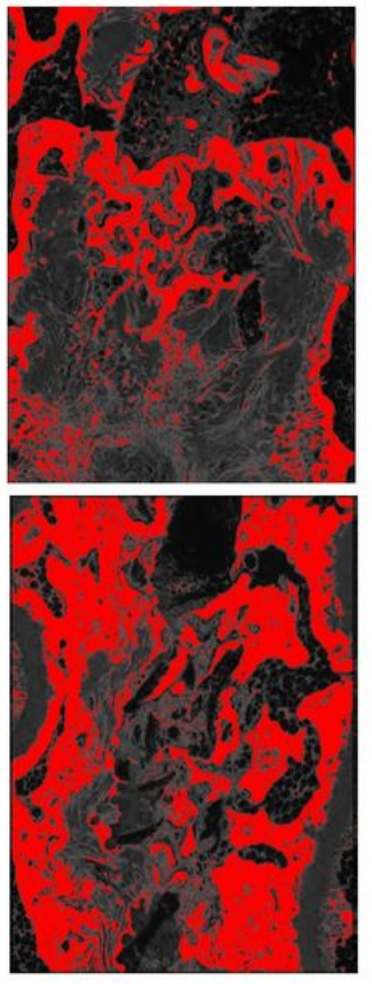

New bone area

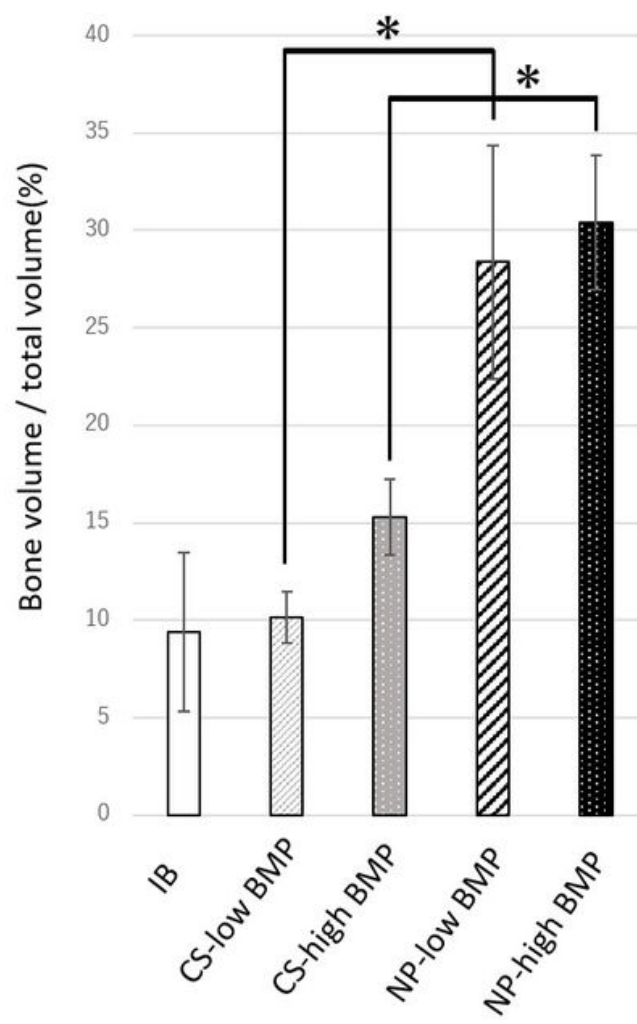

\section{Figure 6}

Quantification of the new bone area in the interbody space. (a) Comparison of new bone area inside the spinal fusion mass in histological sections. A $1.5 \times 2-\mathrm{mm} 2$ ROI (interbody space) was extracted from the newly formed fusion mass. The new bone area (red) was color coded using ImageJ software (version 1.52q, U. S. National Institutes of Health; https://imagej.nih.gov/ij/). (B) The percentage of the newly formed bone area in the ROI was significantly higher in the NP groups than in the CS groups $\left(n=8,{ }^{*}<<\right.$ 0.03) (IB; 9.4\%, CS-low BMP group; 10.1\%, NP-low BMP group; 28.4\%, CS-high group; 15.3\%, NP-high group; $30.4 \%$; data represent mean \pm SD; ${ }^{*} p<0.03$ by Turkey-Krammer test).

(a)

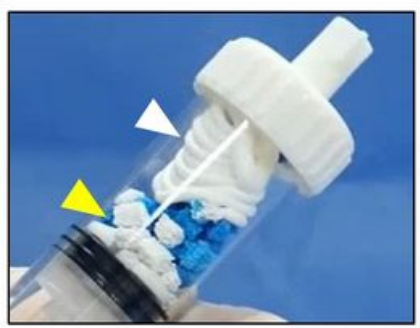

(c)

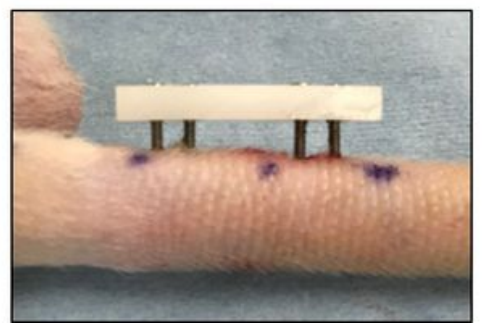

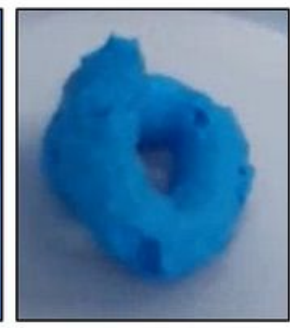

(b)

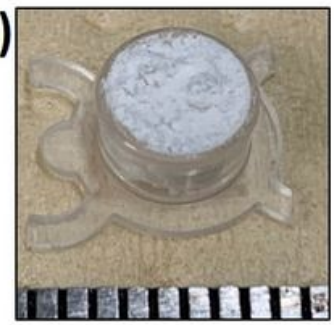

(d)

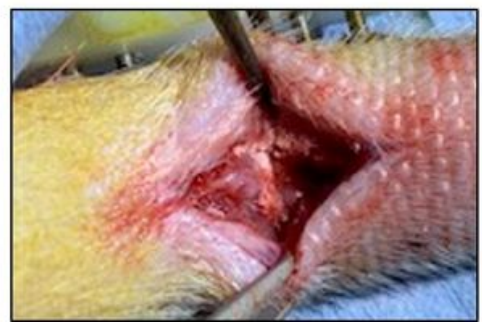

(e)
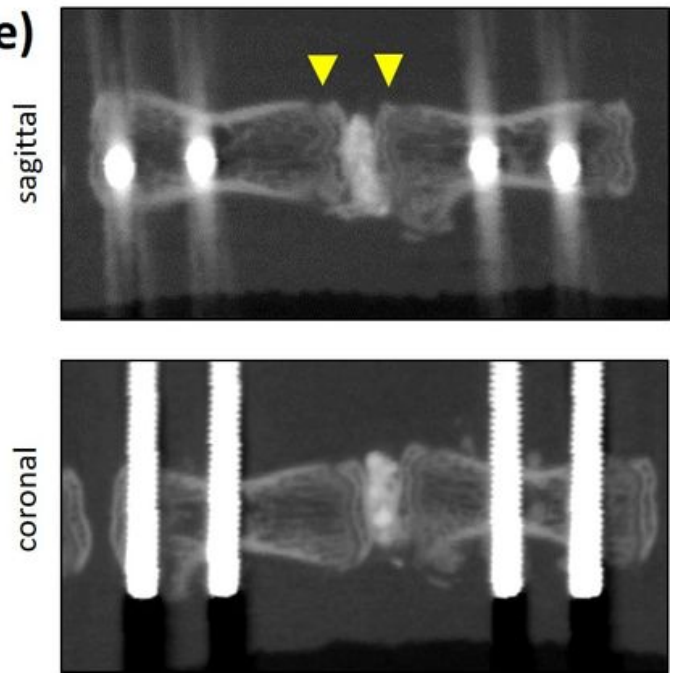


\section{Figure 7}

Production process of HA/ $\beta$-TCP hydrogel, operative photograph, and postoperative micro-computed tomography images.

\section{Supplementary Files}

This is a list of supplementary files associated with this preprint. Click to download.

- Supplementaryinf.pdf 